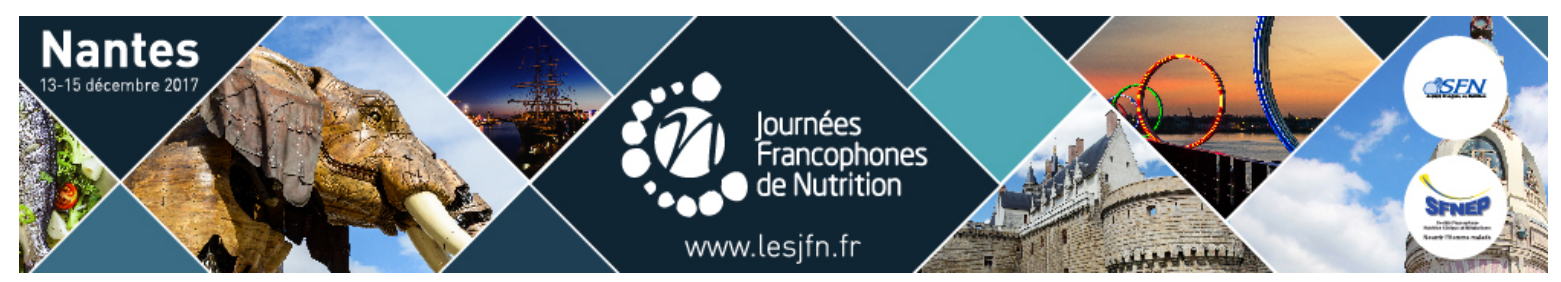

Santé publique

JFN2017/1128

\title{
Identification de biomarqueurs nutritionnels par une approche métabolomique en spectrométrie de masse
}

Céline Dalle1,2, Lucie Lécuyer², 3, Mélanie Petera ${ }^{1}$, Delphine Centeno¹, Bernard Lyan¹, Stéphanie Durand ${ }^{1}$, Estelle PujosGuillot $^{1}$, Pierre Micheau ${ }^{1}$, Christine Morand ${ }^{1}$, Pilar Galan ${ }^{3}$, Serge Hercberg ${ }^{3}, 4$, Valentin Partula ${ }^{3}$, Mélanie Deschasaux ${ }^{3}$,

Bernard Srour ${ }^{3}$, Paule Latino-Martel ${ }^{3}$, Emmanuelle Kesse-Guyot ${ }^{3}$, Mathilde Touvier ${ }^{3}, 5$, Claudine Manach ${ }^{1,5}$

${ }^{1}$ Unité de Nutrition Humaine (UNH), Inra UMR 1019, CRNH, Université Clermont Auvergne, Clermont-Ferrand, ${ }^{2}$

Contribution équivalente (co-premiers), ., ${ }^{3}$ EREN U1153 Inserm / U1125 Inra / Cnam / Université Paris 13 - SMBH, Université Paris 13, Bobigny Cedex, ${ }^{4}$ Département de santé publique, Hôpital Avicenne, Bobigny, ${ }^{5}$ Contribution équivalente (co-derniers), ., France

\section{Discipline: Epidémiologie}

\section{Présentation préférée: Communication orale}

Introduction et but de l'étude: Ce travail s'inscrit dans le cadre du projet Metabo-KS (2015-2017, INCa, PI : M Touvier), qui vise à: découvrir les biomarqueurs prédictifs du cancer du sein, de la qualité du régime alimentaire et d'aliments spécifiques en utilisant la métabolomique, puis à relier ces biomarqueurs pour améliorer la compréhension du rôle de la nutrition sur le risque de cancer du sein. Le présent travail porte sur la découverte de biomarqueurs plasmatiques des apports alimentaires usuels par l'exploration du métabolome alimentaire («food metabolome») par spectrométrie de masse non ciblée.

Matériel et méthodes: Les femmes de la cohorte SU.VI.MAX ayant rempli au moins dix enregistrements alimentaires de 24h ont été stratifiées en déciles selon leur niveau d'adhésion aux recommandations du Programme français National Nutrition Santé. Au total, 80 femmes ont été sélectionnées aléatoirement dans le 10e décile de la distribution PNNS-GS et ont été appariées sur 80 femmes du 1er décile. Les échantillons de plasma prélevés à l'inclusion ont été analysés en spectrométrie de masse non ciblée en utilisant UPLC-ESI-QToF. 1575 et 601 signaux ont été détectés (mode positif et négatif). Les profils métabolomiques ont été comparés en utilisant des méthodes statistiques univariées et multivariées pour sélectionner les ions associés à la consommation de 58 aliments / groupes d'aliments.

Résultats et Analyse statistique : 84 et 30 ions (mode positif et négatif) étaient associés à des aliments spécifiques / des groupes alimentaires ( $r>0,3$, valeur $\mathrm{p}$ avec $\mathrm{BH}<0,1)$. Certains d'entre eux avaient été suggérés précédemment comme biomarqueurs dans des études de métabolomique, comme la proline-bétaïne pour la consommation d'orange. Cela démontre la pertinence de notre stratégie. L'identification des autres biomarqueurs candidats est en cours (grâce à une base de données en ligne, à la littérature et à la méthode LC-MS / MS sur LTQ-OrbiTrap).

Conclusion: Ce travail contribuera à fournir de nouveaux biomarqueurs de l'apport alimentaire qui seront utiles pour améliorer la qualité de l'évaluation des expositions nutritionnelles dans les études épidémiologiques.

Remerciements: Institut National du Cancer (INCa) / Institut Fédératif de Recherche biomédicale (IFRB) / Canceropôle Ile-deFrance / Projet labellisé NACRe Partenariat

Conflits d'intérêts: Aucun conflit à déclarer 